\title{
AUTOMATIC CO-REGISTRATION OF MULTI-TEMPORAL LANDSAT-8/OLI AND SENTINEL-2A/MSI IMAGES
}

\author{
S. Skakun ${ }^{1,2}$, J.-C. Roger ${ }^{1,2}$, E. Vermote ${ }^{2}$, \\ C. Justice ${ }^{1}$, J. Masek ${ }^{3}$
}

${ }^{1}$ Department of Geographical Sciences, University of Maryland, College Park MD 20742, USA

2 NASA Goddard Space Flight Center Code 619, Greenbelt, MD 20771, USA

${ }^{3}$ NASA Goddard Space Flight Center Code 618, Greenbelt, MD 20771, USA 


\section{Introduction}

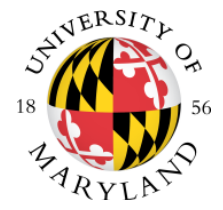

- Many applications in climate change and environmental and agricultural monitoring rely heavily on the exploitation of multi-temporal satellite imagery

- Combined use of freely available Landsat-8 and Sentinel-2 images can offer high temporal frequency of about 1 image every $3-5$ days globally

- Data should be consistent

- Including co-registration

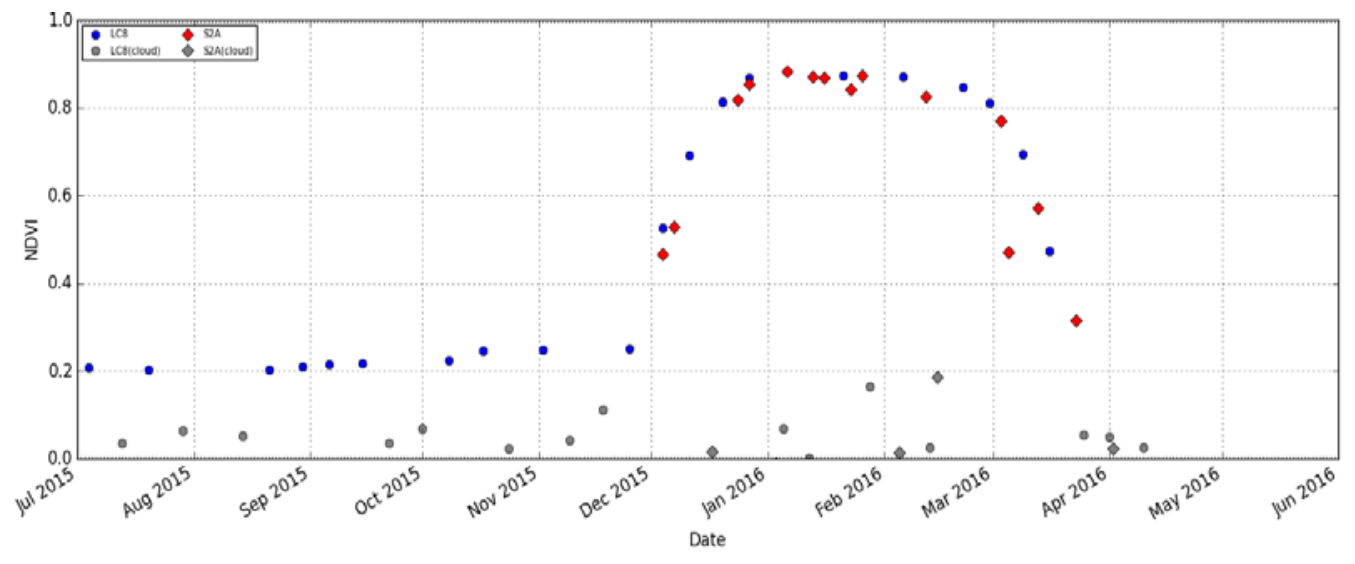




\section{Introduction: Sentinel-2A/MSI}

- $\mathrm{MSI}=$ Multi-Spectral Instrument

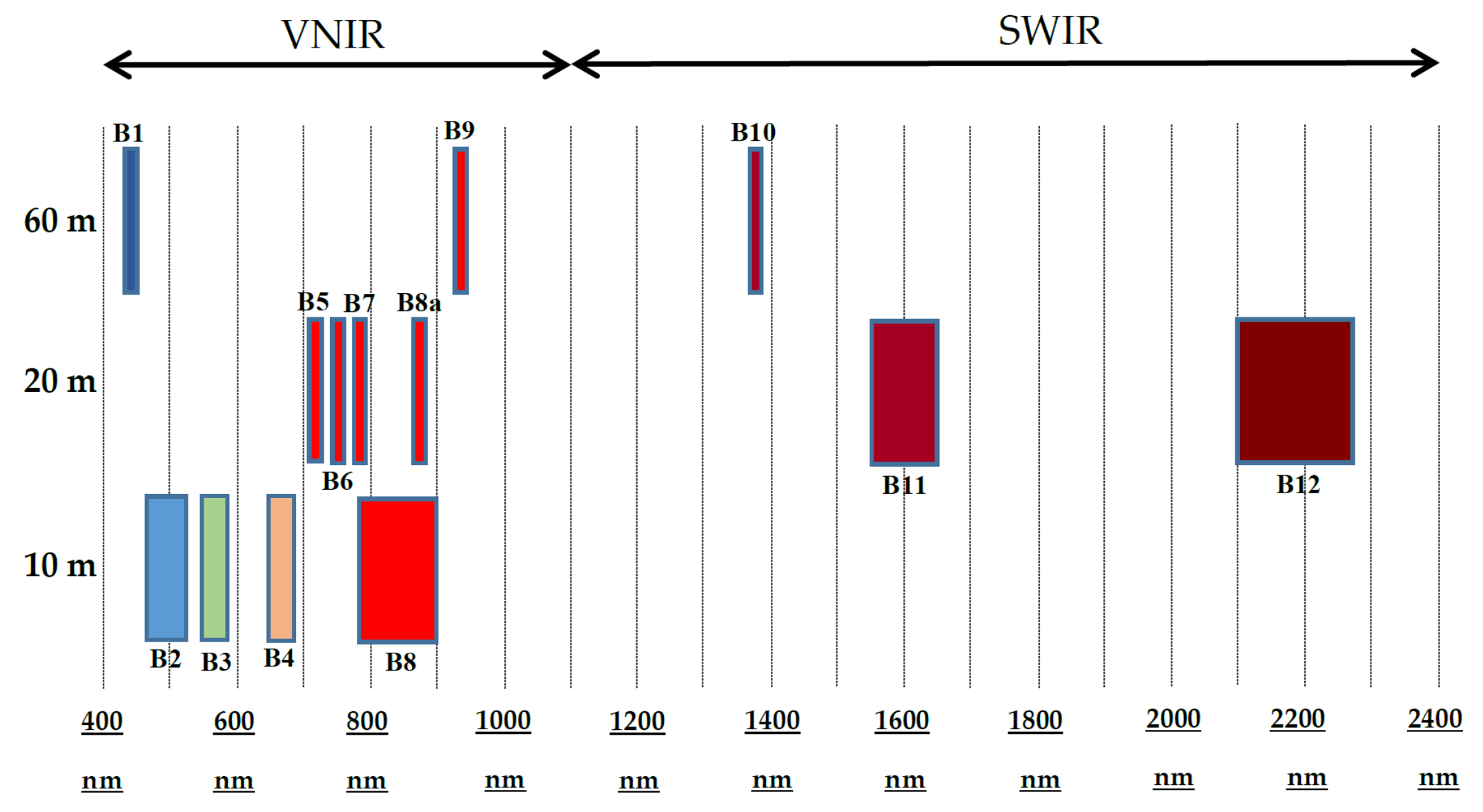

(Gascon et al. 2017) 


\section{Introduction: Landsat-8}

- Operational Land Imager (OLI) and Thermal Infrared Sensor (TIRS) instruments

Comparison of Landsat 7 and 8 bands with Sentinel-2

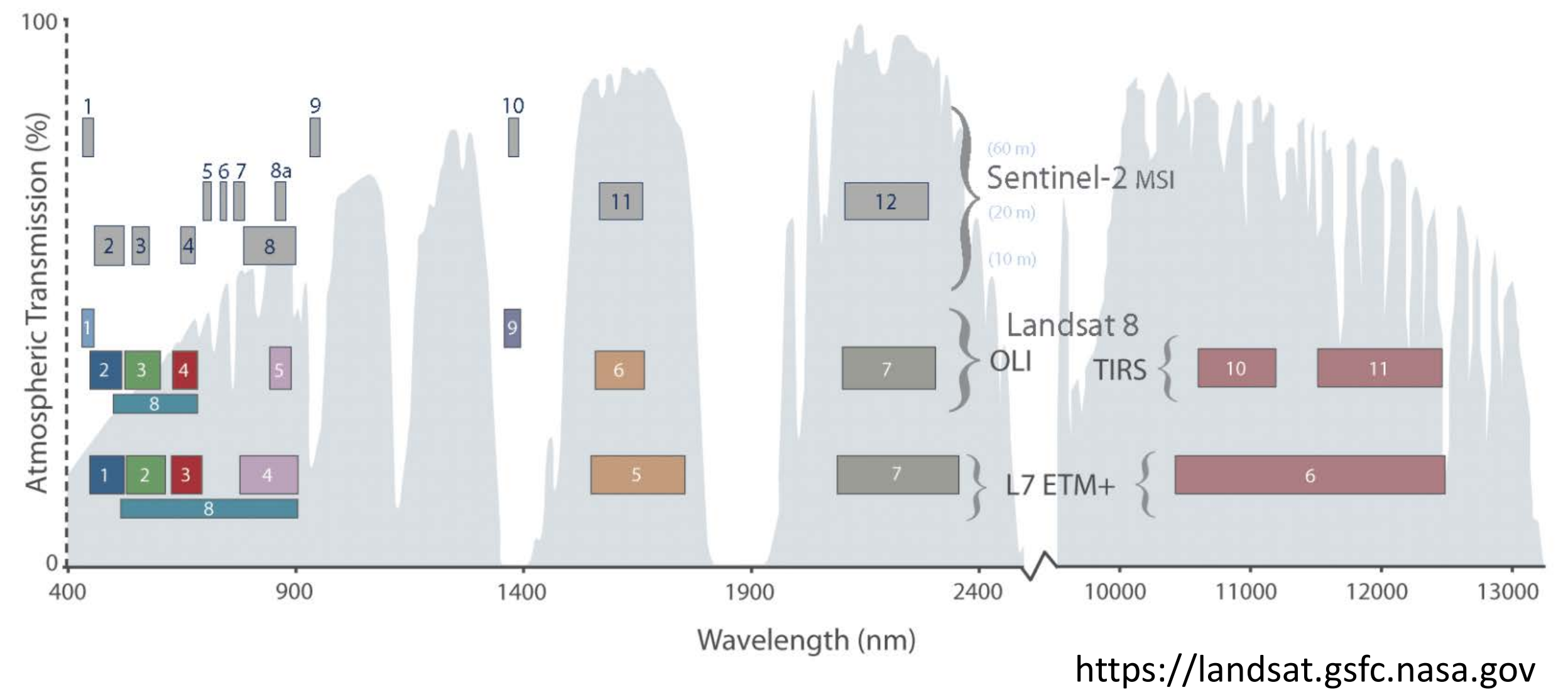




\section{Introduction}

- Both sensor geolocation systems are designed to use ground control to improve the geolocation accuracy and repeatability (Storey et al. 2016)

- Sentinel-2A

- The Sentinel-2 geolocation will use a Global Reference Image (GRI) derived from orthorectified Sentinel-2 cloud-free images (Déchoz et al. 2015)

- Planned to be available at the end of 2017

- Landsat-8

- The Landsat-8 geolocation uses a global sample of ground control points (Storey et al., 2014) derived for each WRS-2 path/row of circa 2000 Global Land Survey (GLS) Landsat-7 imagery (Gutman et al., 2013).

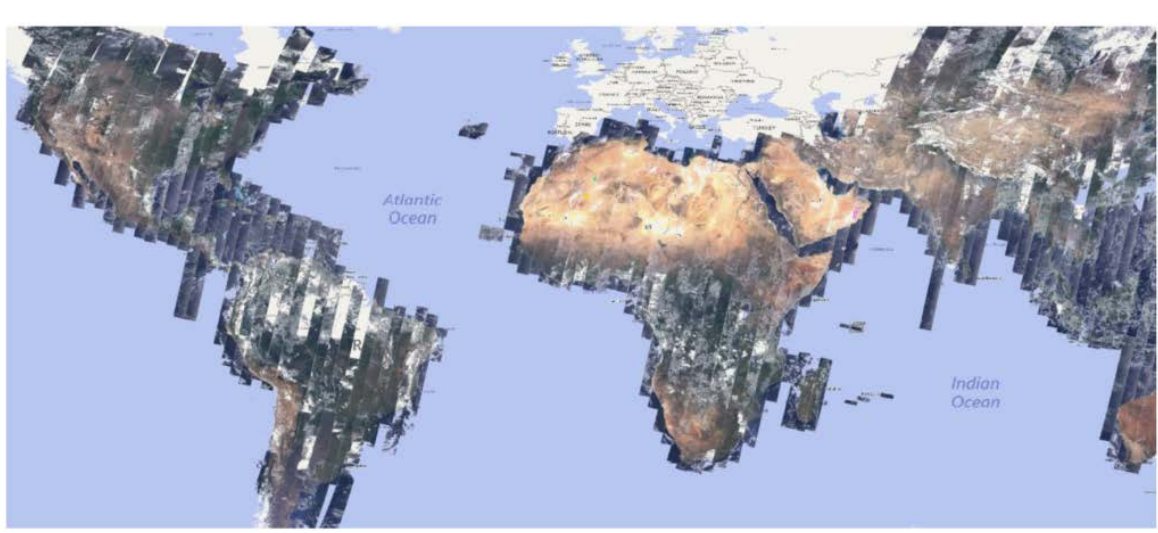

Figure 17. Overview of the Sentinel-2 GRI selection, July 2016 (European GRI products are not present on this map, since they were produced in the In-Orbit Commissioning Review (IOCR) context, in October 2015)

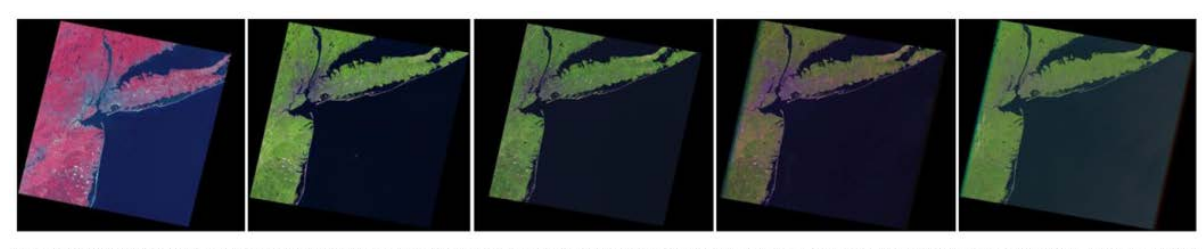
These images of New York City, NewY https://landsat.usgs.gov/global-land-surveys-gls 


\section{Landsat-8/Sentinel-2A Harmonization}

L8 $30 \mathrm{~m}$ pixel

- Pixel value misalignment

- LC8 (center) and S2 (UL)

- Different UTM zones:

- L8 uses north zone even for southern hemisphere, while S2 uses south zones

- e.g. $20 \mathrm{~N}$ from LC8 vs

$20 S$ from S2

- Misregistration

- "estimate of the expected Sentinel-2 to Landsat-8

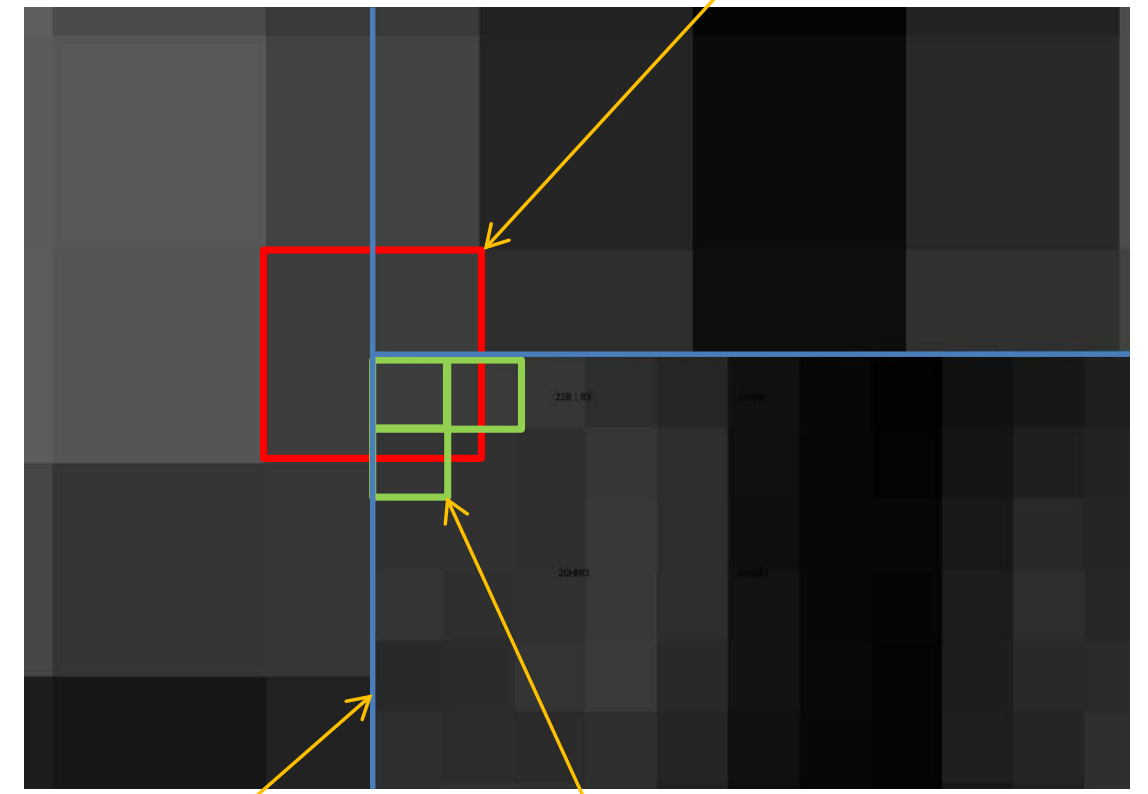
misregistration .... yields a 38 meter $(2 \sigma)$ expected registration accuracy between the sensors" [Storey et al., RSE, 2016]

S2 tile boundary $\quad$ S2 $10 \mathrm{~m}$ pixel 


\section{Landsat-8/Sentinel-2A Misregistration}

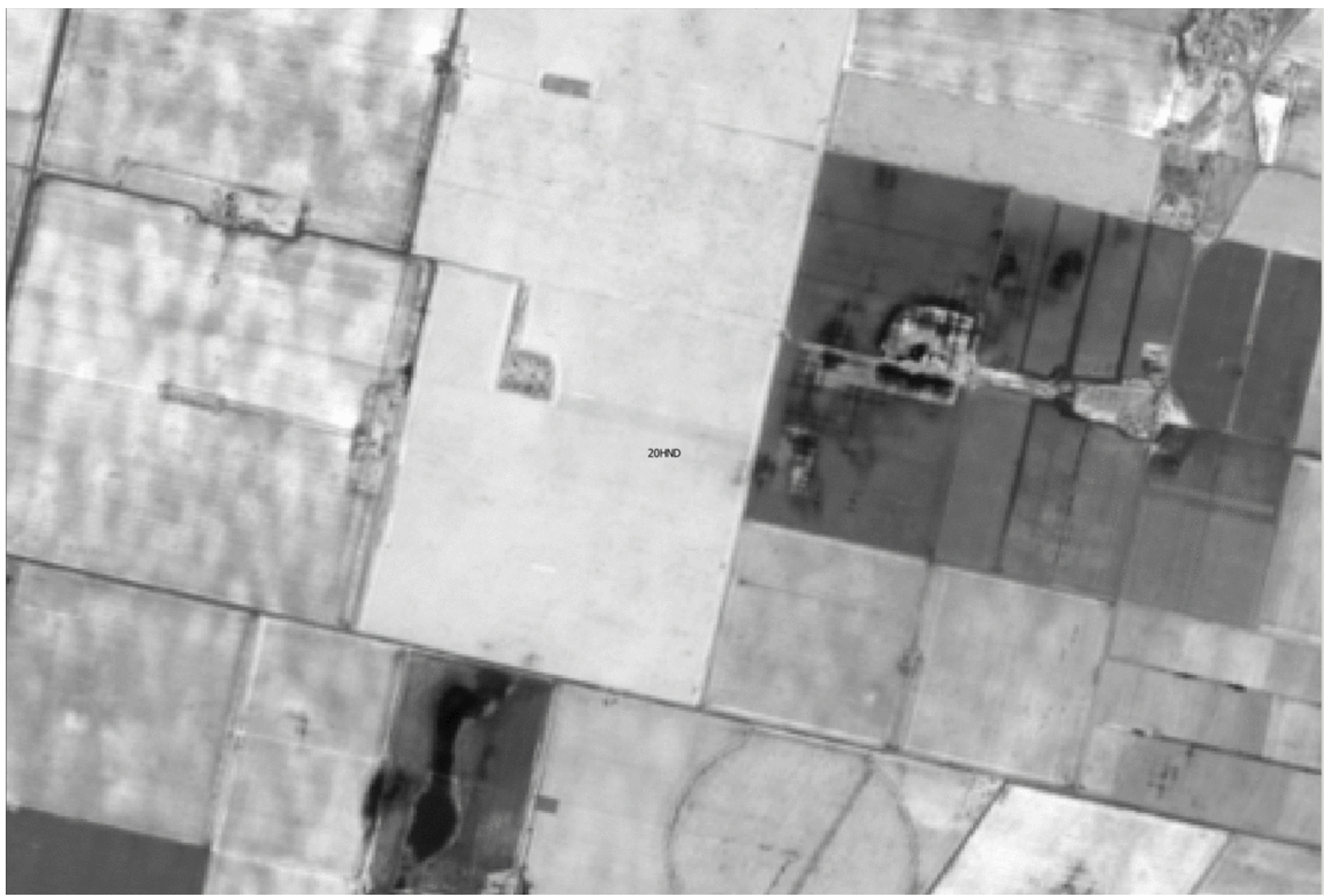

T20HNH - Sentinel-2A, band 08 (NIR), $10 \mathrm{~m}$ - Landsat-8, band5 (NIR), $30 \mathrm{~m}$ 


\section{Methodology}

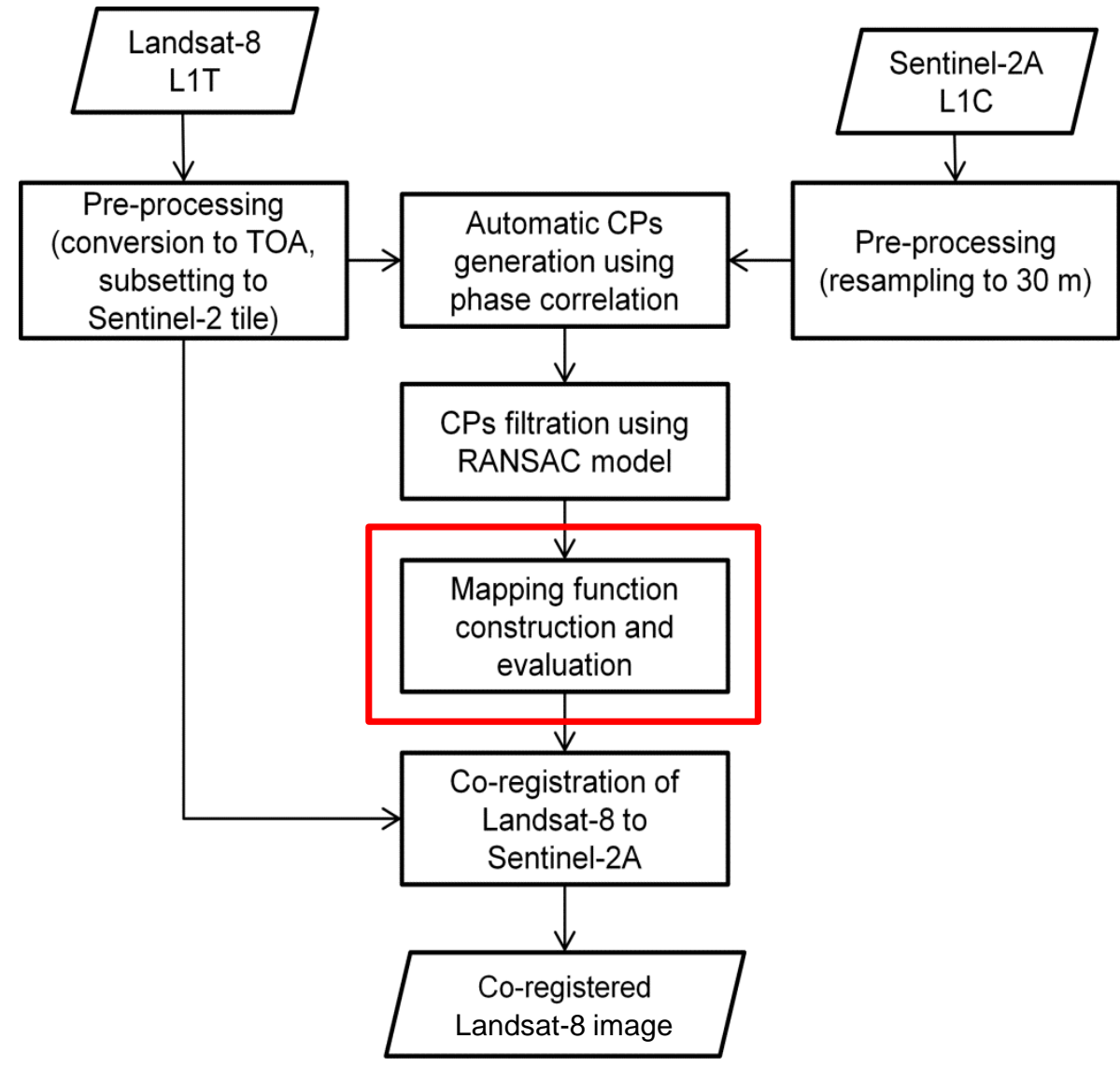

S. Skakun, J.-C. Roger, E. F. Vermote, J. G. Masek, and C. O. Justice, "Automatic sub-pixel co-registration of Landsat-8 Operational Land Imager and Sentinel-2A Multi-Spectral Instrument images using phase correlation and machine learning based mapping," Int. J. Digital Earth, 2017, doi:10.1080/17538947.2017.1304586.
- Transformation function.

- A transformation function $F()$ is built to find correspondence between CPs in the reference image $\mathbf{x}_{r}=\left(x_{r}, y_{r}\right)$ and points in the sensed image $\mathbf{x}_{s}=\left(x_{s}, y_{s}\right)$ : $\left(x_{s}, y_{s}\right)=F\left(x_{r}, y_{r}\right)$.

- The following functions are evaluated:

- Polynomial

- Radial Basis Functions (RBFs)

- Gaussian

- Thin-plate splines (TPS)

- Random Forest (RF) regression 


\section{Data used}

\section{- Co-registration of $\mathbf{4 5}$ Landsat-8 to Sentinel-2A pairs and 37 Sentinel-2A to Sentinel-2A pairs were analyzed.}

Table 1. Description of data used in the study.

\begin{tabular}{|c|c|c|c|c|}
\hline Country & $\begin{array}{c}\text { Tile } \\
\text { number }\end{array}$ & $\begin{array}{l}\text { Acquisition date of } \\
\text { Sentinel-2A } \\
\text { reference image }\end{array}$ & $\begin{array}{l}\text { Acquisition dates of Landsat- } 8 \text { co- } \\
\text { registered images }\end{array}$ & $\begin{array}{l}\text { Acquisition dates of Sentinel-2A co- } \\
\text { registered images }\end{array}$ \\
\hline Argentina & $20 \mathrm{HNH}$ & 2015358 & $\begin{array}{l}\text { 2015354, 2015185, 2015201, 2015242, } \\
\text { 2015249, 2015258, 2015290, 2015306, } \\
\text { 2015329, 2015338, 2015345, 2015361, } \\
\text { 2016021, 2016037, 2016053 }\end{array}$ & $\begin{array}{l}\text { 2015341, 2016006, 2016013, 2016016, } \\
\text { 2016023, 2016026, 2016036, 2016043, } \\
\text { 2016046, 2016063, 2016065, 2016073, } \\
\text { 2016083, 2016093, 2016096 }\end{array}$ \\
\hline Argentina & $20 \mathrm{HPH}$ & 2015358 & $\begin{array}{l}\text { 2015242, 2015258, 2015290, 2015306, } \\
2015338,2015354,2016021,2016037 \\
2016053\end{array}$ & $\begin{array}{r}2016003,2016013,2016023,2016043 \\
2016063,2016073,2016083,2016093\end{array}$ \\
\hline $\begin{array}{l}\text { US } \\
\text { (Texas) }\end{array}$ & 14SKF & 2016012 & $\begin{array}{l}\text { 2015245, 2015261, 2015293, 2015309, } \\
\text { 2015325, 2015341, 2015357, 2016024, } \\
\text { 2016040, 2016056, 2016072, 2016088, } \\
2016104\end{array}$ & 2016042, 2016072, 2016132 \\
\hline Ukraine & 36UUU & 2016169 & $\begin{array}{l}\text { 2016076, 2016092, 2016108, 2016156, } \\
\text { 2016172, 2016188 }\end{array}$ & $\begin{array}{l}\text { 2016096, 2016109, 2016119, 2016156, } \\
\text { 2016166, 2016179, 2016196, 2016199, } \\
2016206\end{array}$ \\
\hline Ukraine & 34UFU & 2016198 & 2016063,2016182 & 2016048,2016208 \\
\hline
\end{tabular}

Note: Acquisition dates are given in the format YY YY DOY (where DOY is the day of the year). 


\section{Results}
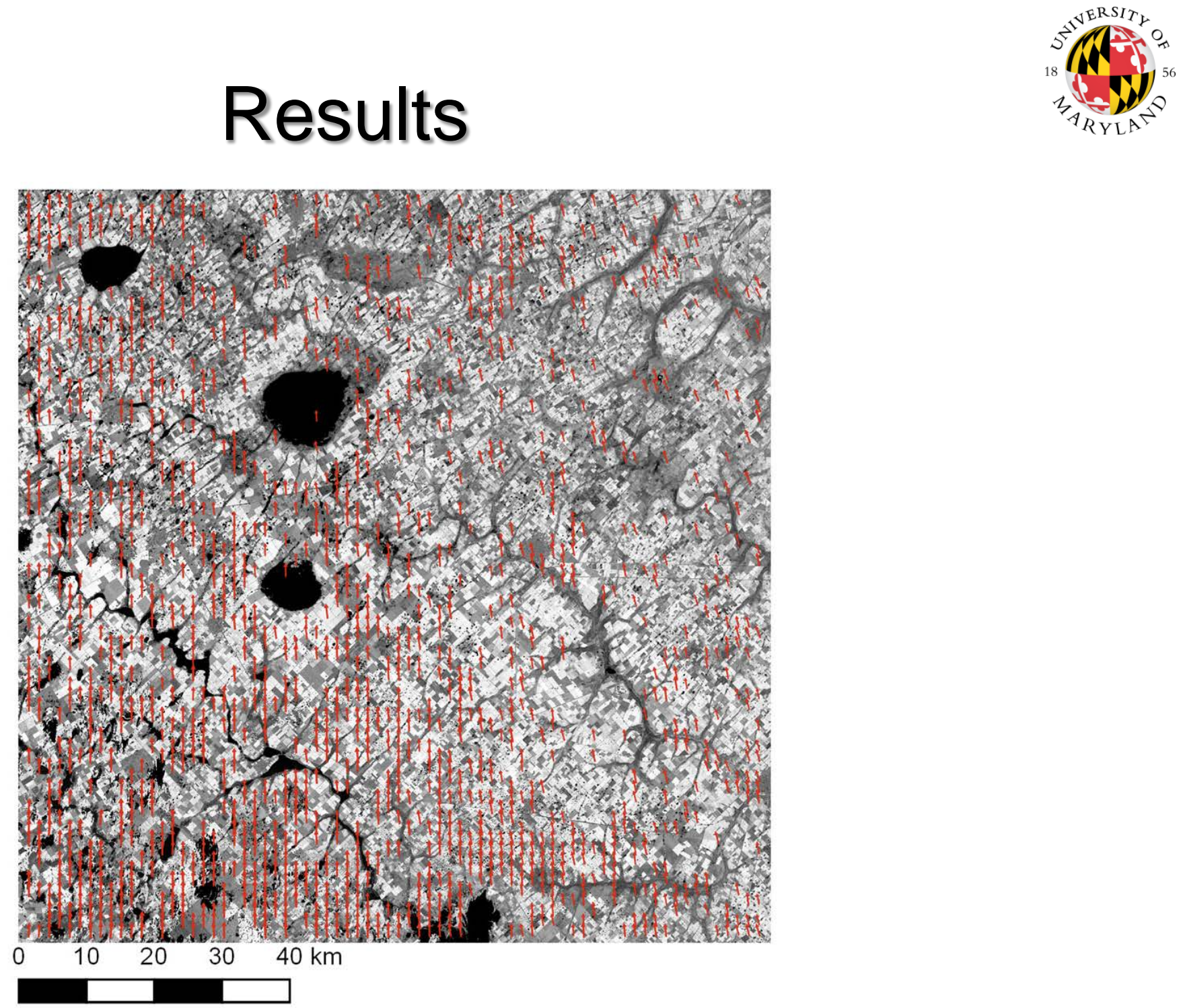

Figure 3. Location of CPs shown in the form of vectors outlining the direction and magnitude of shifts $\left(\Delta_{x}\right.$ and $\Delta_{y}$ (Equation (2)) found between Landsat-8 image acquired on 2016021 (21-Jan-2016), and Sentinel-2A image acquired on 2015358 (24-Dec-2015) and used as a reference image, over the study area in Argentina, tile T2OHNH. Vector lengths were multiplied by 100 for visual clarity. Overall, 1634 CPs were found using the phase-correlation approach in this case. The background is a Landsat-8 TOA NIR (band 5) image with TOA reflectance values scaled from 0.05 to 0.65 . 


\section{Results}
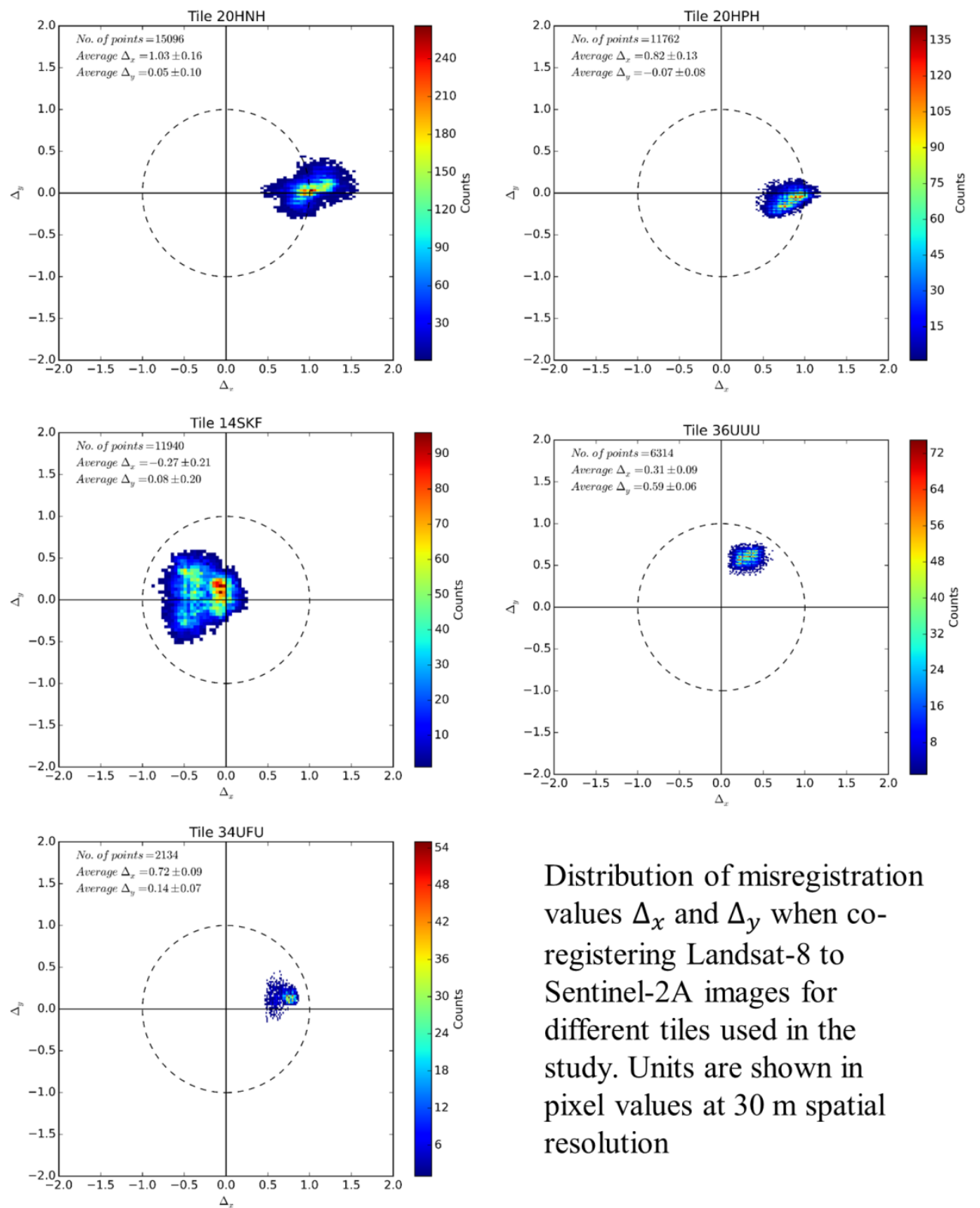

Distribution of misregistration values $\Delta_{x}$ and $\Delta_{y}$ when coregistering Landsat-8 to Sentinel-2A images for different tiles used in the study. Units are shown in pixel values at $30 \mathrm{~m}$ spatial resolution 


\section{Results}
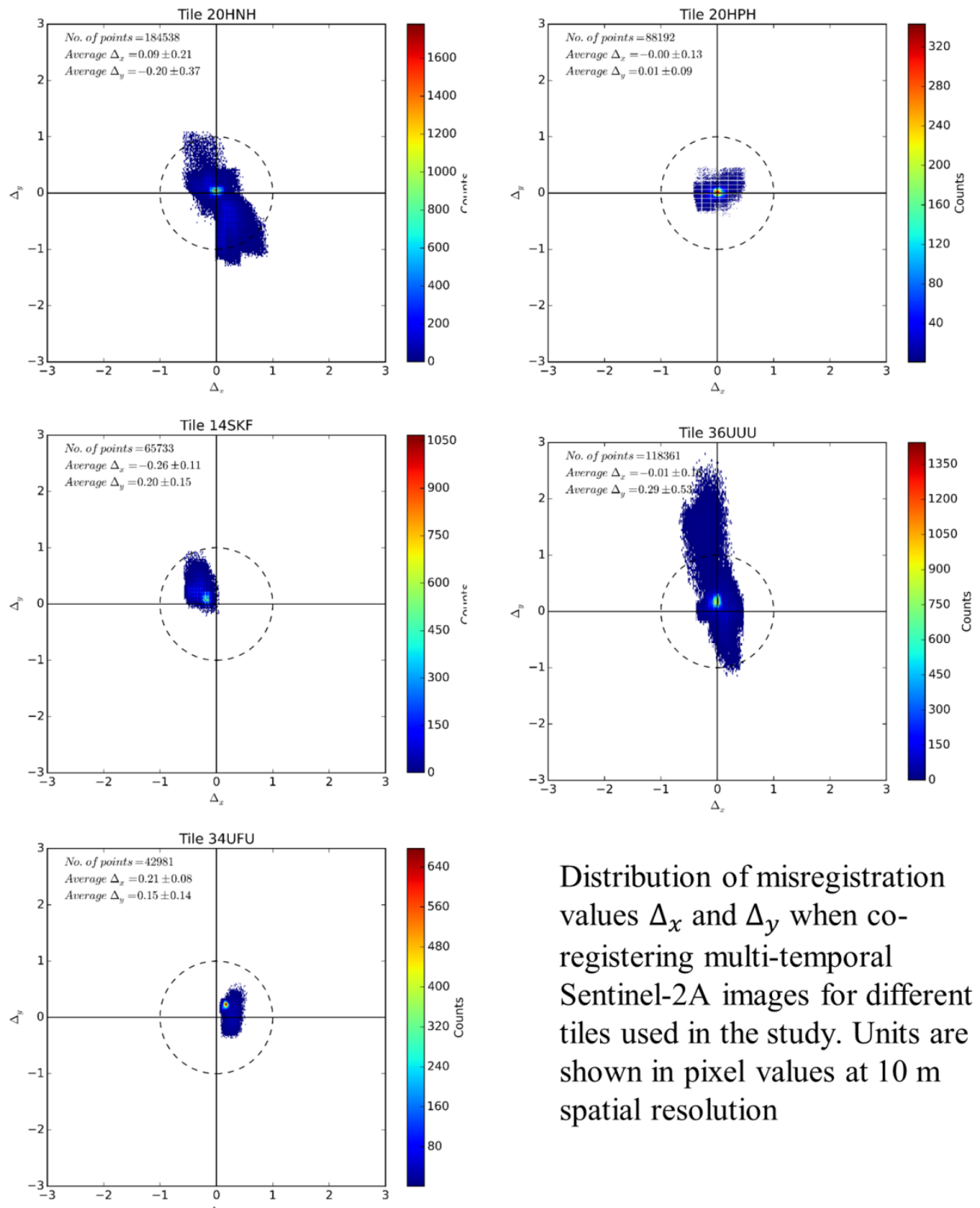

Distribution of misregistration values $\Delta_{x}$ and $\Delta_{y}$ when coregistering multi-temporal Sentinel-2A images for different tiles used in the study. Units are shown in pixel values at $10 \mathrm{~m}$ spatial resolution 


\section{Results}

\section{- Performance of different transformation functions when co- registering Landsat-8 to Sentinel-2A at $30 \mathrm{~m}$}

Table 4. Average and standard deviation of the RMSE error (Equation (4)) calculated for different transformation functions using CPs from testing set when co-registering Landsat-8 and Sentinel-2A images.

\begin{tabular}{|c|c|c|c|c|c|c|c|c|c|c|}
\hline \multirow[b]{2}{*}{ Tile } & \multicolumn{2}{|c|}{$\begin{array}{c}\text { Translation } \\
\text { (Equations (7)-(8)) }\end{array}$} & \multicolumn{2}{|c|}{$\begin{array}{l}\text { 1st order polynomial } \\
\text { (Equations (9)-(10)) }\end{array}$} & \multicolumn{2}{|c|}{$\begin{array}{c}\text { Gaussian RBFs } \\
\text { (Equations (11)-(12), } \\
(13))\end{array}$} & \multicolumn{2}{|c|}{ RF regression } & \multicolumn{2}{|c|}{$\begin{array}{l}\text { TPS (Equations (11)- } \\
(12),(14))\end{array}$} \\
\hline & Mean & $\begin{array}{l}\text { Standard } \\
\text { deviation }\end{array}$ & Mean & $\begin{array}{l}\text { Standard } \\
\text { deviation }\end{array}$ & Mean & $\begin{array}{l}\text { Standard } \\
\text { deviation }\end{array}$ & Mean & $\begin{array}{l}\text { Standard } \\
\text { deviation }\end{array}$ & Mean & $\begin{array}{l}\text { Standard } \\
\text { deviation }\end{array}$ \\
\hline $20 \mathrm{HNH}$ & 0.119 & 0.031 & 0.091 & 0.026 & 0.093 & 0.027 & 0.084 & 0.024 & 0.090 & 0.025 \\
\hline $20 \mathrm{HPH}$ & 0.123 & 0.014 & 0.078 & 0.016 & 0.081 & 0.017 & 0.073 & 0.018 & 0.079 & 0.018 \\
\hline 36UUU & 0.108 & 0.011 & 0.072 & 0.015 & 0.074 & 0.015 & 0.059 & 0.014 & 0.073 & 0.015 \\
\hline 14SKF & 0.145 & 0.037 & 0.094 & 0.018 & 0.095 & 0.018 & 0.074 & 0.018 & 0.094 & 0.017 \\
\hline 34UFU & 0.095 & 0.045 & 0.056 & 0.034 & 0.060 & 0.038 & 0.044 & 0.030 & 0.054 & 0.033 \\
\hline
\end{tabular}

Note: RMSE values are shown in pixel units at $30 \mathrm{~m}$ spatial resolution. 
- Performance of different transformation functions when coregistering Sentinel-2A to Sentinel-2A at $10 \mathrm{~m}$

Table 5. Average and standard deviation of the RMSE error (Equation (4)) calculated for different transformation functions using CPs from testing set when co-registering multi-temporal Sentinel-2A images from the same orbit.

\begin{tabular}{|c|c|c|c|c|c|c|c|c|c|c|}
\hline \multirow[b]{2}{*}{ Tile } & \multicolumn{2}{|c|}{$\begin{array}{c}\text { Translation } \\
\text { (Equations (7)-(8)) }\end{array}$} & \multicolumn{2}{|c|}{$\begin{array}{l}\text { 1st order polynomial } \\
\text { (Equations (9)-(10)) }\end{array}$} & \multicolumn{2}{|c|}{$\begin{array}{c}\text { Gaussian RBFs } \\
\text { (Equations (11)-(12), } \\
(13)) \\
\end{array}$} & \multicolumn{2}{|c|}{ RF regression } & \multicolumn{2}{|c|}{$\begin{array}{c}\text { TPS (Equations (11)- } \\
(12),(14))\end{array}$} \\
\hline & Mean & $\begin{array}{l}\text { Standard } \\
\text { deviation }\end{array}$ & Mean & $\begin{array}{l}\text { Standard } \\
\text { deviation }\end{array}$ & Mean & $\begin{array}{l}\text { Standard } \\
\text { deviation }\end{array}$ & Mean & $\begin{array}{l}\text { Standard } \\
\text { deviation }\end{array}$ & Mean & $\begin{array}{l}\text { Standard } \\
\text { deviation }\end{array}$ \\
\hline $20 \mathrm{HNH}$ & 0.141 & 0.104 & 0.125 & 0.076 & 0.125 & 0.076 & 0.105 & 0.060 & 0.126 & 0.074 \\
\hline $20 \mathrm{HPH}$ & 0.133 & 0.064 & 0.128 & 0.062 & 0.127 & 0.062 & 0.114 & 0.059 & 0.129 & 0.064 \\
\hline $36 U U U$ & 0.181 & 0.126 & 0.114 & 0.046 & 0.114 & 0.046 & 0.088 & 0.035 & 0.112 & 0.048 \\
\hline 14SKF & 0.133 & 0.066 & 0.123 & 0.050 & 0.118 & 0.046 & 0.089 & 0.036 & 0.123 & 0.051 \\
\hline 34UFU & 0.122 & 0.101 & 0.092 & 0.086 & 0.091 & 0.084 & 0.066 & 0.059 & 0.093 & 0.088 \\
\hline
\end{tabular}

Note: RMSE values are shown in pixel units at $10 \mathrm{~m}$ spatial resolution.

Table 6. The same as Table 5, but for adjacent Sentinel-2A orbits.

\begin{tabular}{|c|c|c|c|c|c|c|c|c|c|c|}
\hline \multirow[b]{2}{*}{ Tile } & \multicolumn{2}{|c|}{$\begin{array}{c}\text { Translation } \\
\text { (Equations (7)-(8)) } \\
\end{array}$} & \multicolumn{2}{|c|}{$\begin{array}{l}\text { 1st order polynomial } \\
\text { (Equations (9)-(10)) }\end{array}$} & \multicolumn{2}{|c|}{$\begin{array}{c}\text { Gaussian RBFs } \\
\text { (Equations (11)-(12), } \\
(13)) \\
\end{array}$} & \multicolumn{2}{|c|}{ RF regression } & \multicolumn{2}{|c|}{$\begin{array}{c}\text { TPS (Equations (11) } \\
(12),(14))\end{array}$} \\
\hline & Mean & $\begin{array}{l}\text { Standard } \\
\text { deviation }\end{array}$ & Mean & $\begin{array}{l}\text { Standard } \\
\text { deviation }\end{array}$ & Mean & $\begin{array}{l}\text { Standard } \\
\text { deviation }\end{array}$ & Mean & $\begin{array}{l}\text { Standard } \\
\text { deviation }\end{array}$ & Mean & $\begin{array}{l}\text { Standard } \\
\text { deviation }\end{array}$ \\
\hline $20 \mathrm{HNH}$ & 0.239 & 0.048 & 0.207 & 0.031 & 0.202 & 0.028 & 0.164 & 0.032 & 0.205 & 0.031 \\
\hline $36 U U U$ & 0.248 & 0.212 & 0.191 & 0.139 & 0.189 & 0.138 & 0.138 & 0.087 & 0.193 & 0.142 \\
\hline
\end{tabular}




\section{Results}

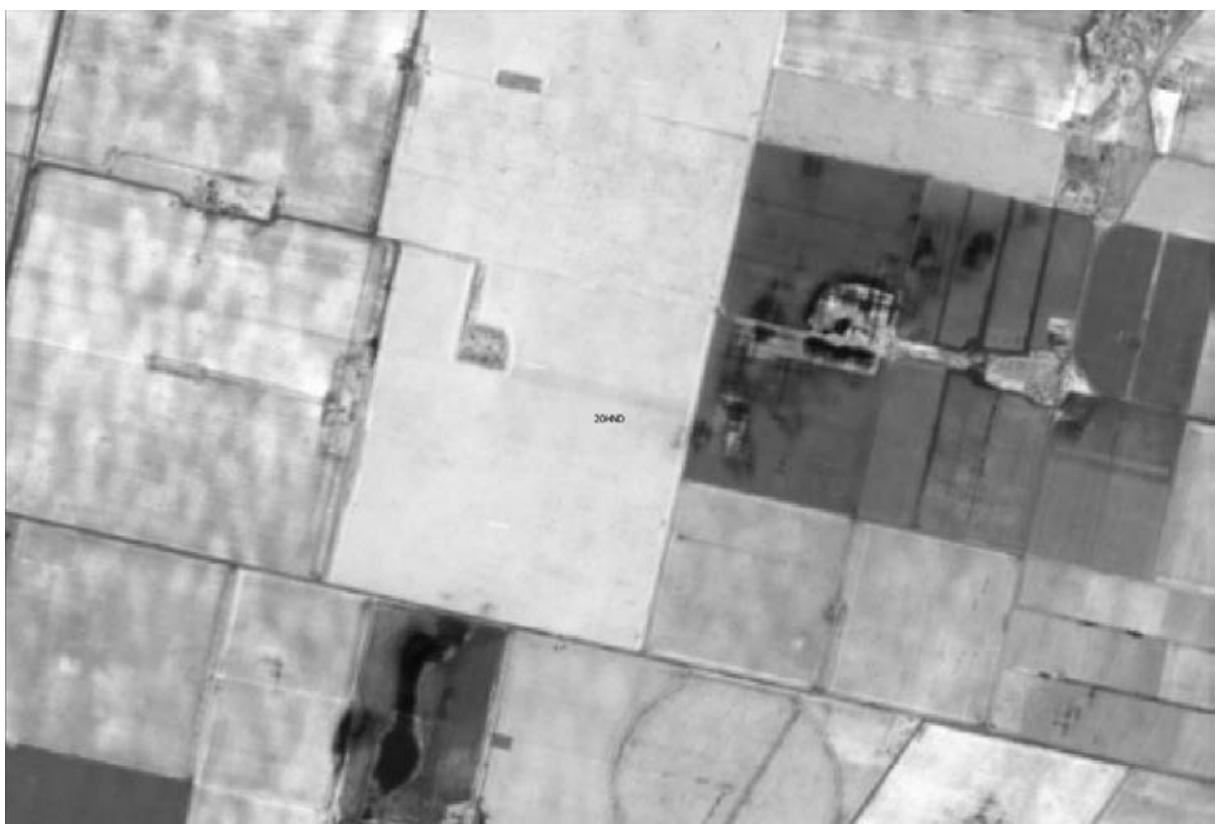

Without co-registration

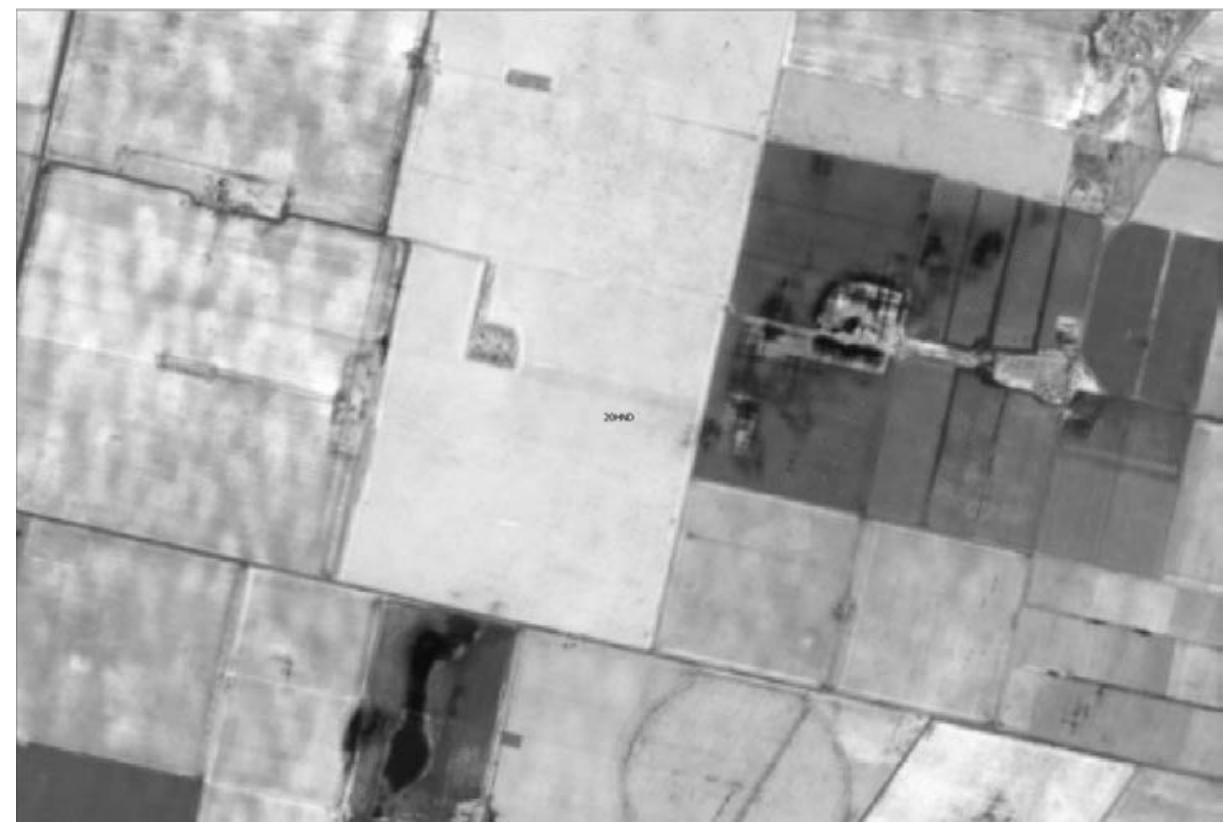

With co-registration

T20HNH - Sentinel-2A, band 08 (NIR), $10 \mathrm{~m}$ - Landsat-8, band5 (NIR), $30 \mathrm{~m}$ 


\section{Results}
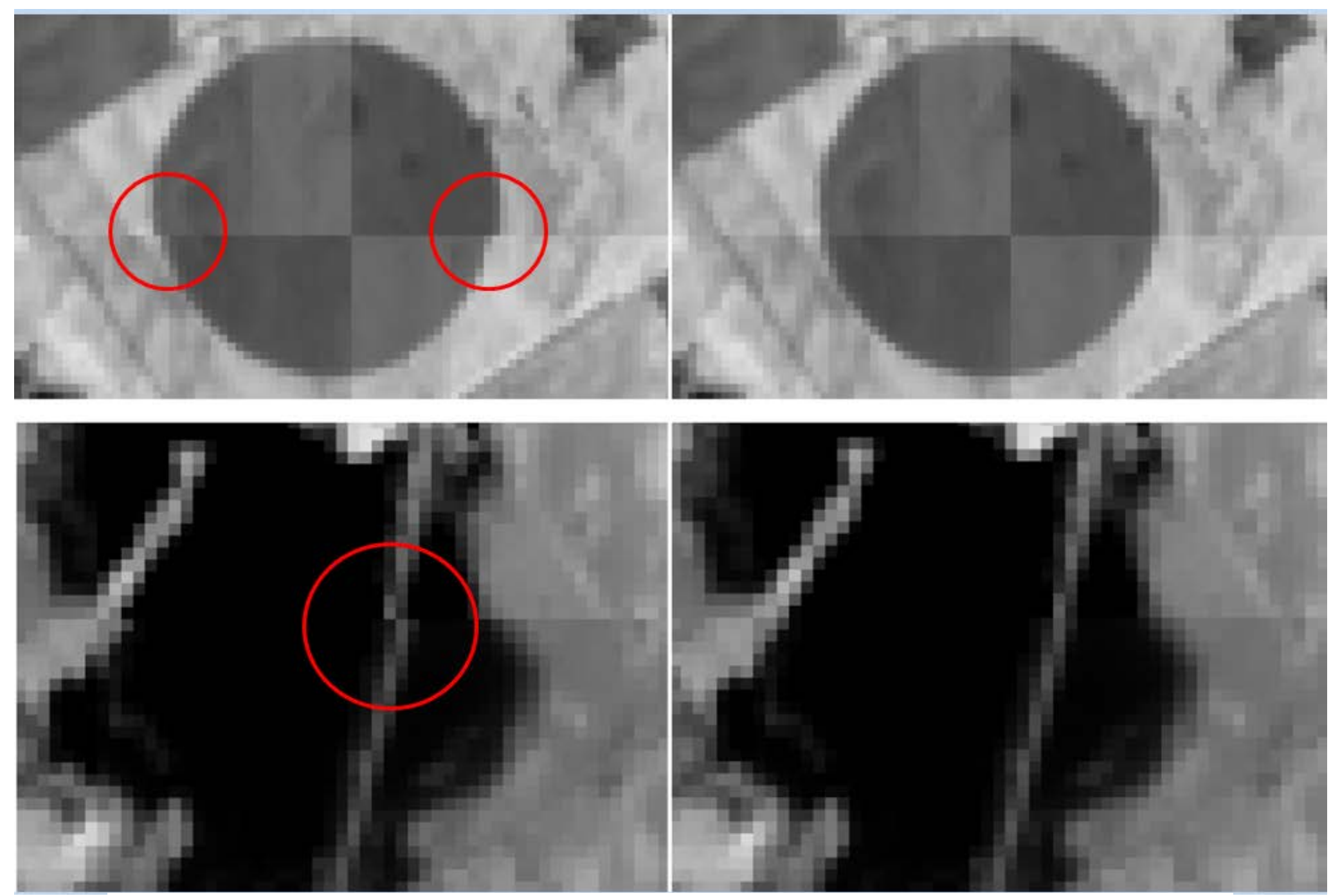

A 30 m “chessboard” composed of alternating Landsat-8 (acquired on 20-Dec-2015) and Sentinel-2A (24-Dec-2015) images before (left) and after co-registration (right). 


\section{Sentinel-2A Multi-spectral Misregistration}

\begin{tabular}{|c|c|c|c|c|c|c|c|c|c|c|c|c|c|}
\hline \multicolumn{10}{|c|}{$\mathrm{B} / \mathrm{H}=0.018$} & \multicolumn{4}{|c|}{$\mathrm{B} / \mathrm{H}=0.010$} \\
\hline Band & 2 & 8 & 3 & 4 & 5 & 6 & 7 & $8 a$ & 1 & 9 & 10 & 11 & 12 \\
\hline \multirow[t]{3}{*}{$\begin{array}{c}\text { Odd/even detector } \\
\text { parallax } \\
\text { angle }(\mathrm{B} / \mathrm{H})\end{array}$} & 0,022 & 0,026 & 0,030 & 0,034 & 0,038 & 0,042 & 0,046 & 0,051 & 0,055 & 0,059 & 0,030 & 0,040 & 0,050 \\
\hline & \multicolumn{4}{|c|}{$10 \mathrm{~m}$} & \multicolumn{4}{|c|}{$20 \mathrm{~m}$} & \multicolumn{2}{|c|}{$60 \mathrm{~m}$} & & & \\
\hline & & & & & VNI & & & & & & & & \\
\hline
\end{tabular}

Figure 2: Staggered detector configuration and inter-detector/inter-band parallax angles

[Sentinel-2 Products Specification Document https://sentinel.esa.int/documents/ 247904/685211/Sentinel-2-ProductSpecifications-Document] 


\section{Sentinel-2A Multi-spectral Misregistration}

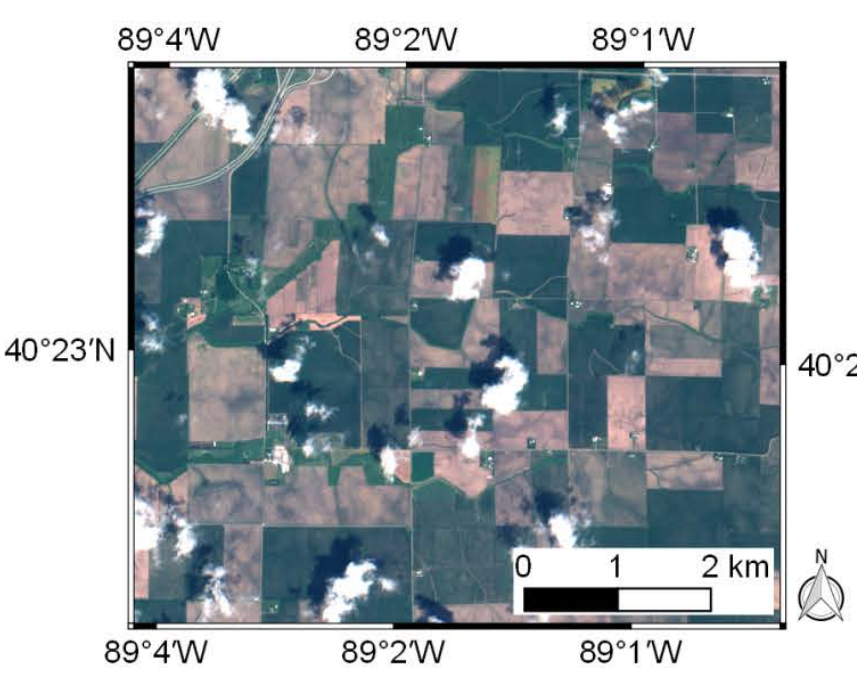

(a)

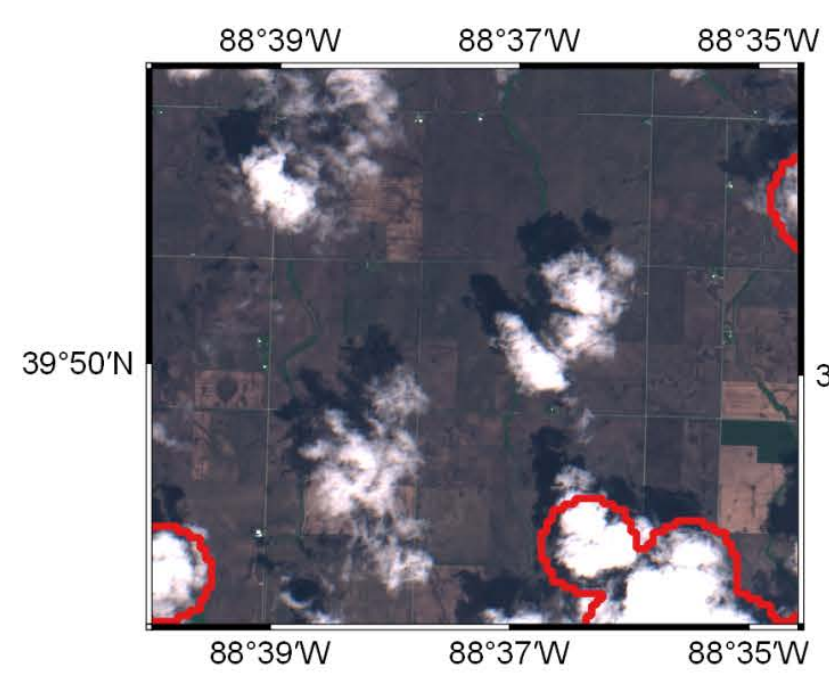

(d)

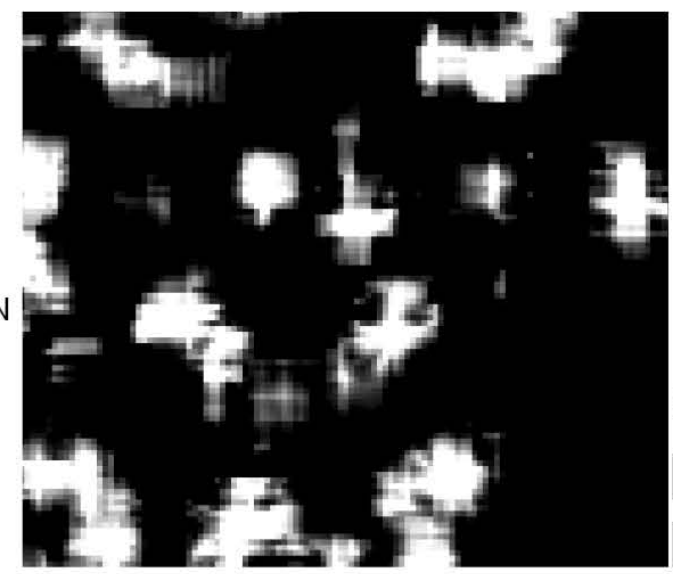

(b)

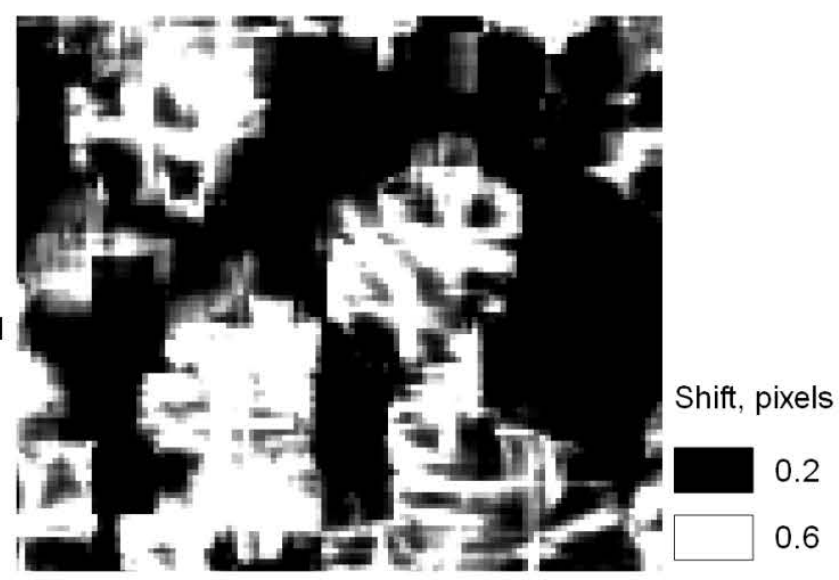

(e)

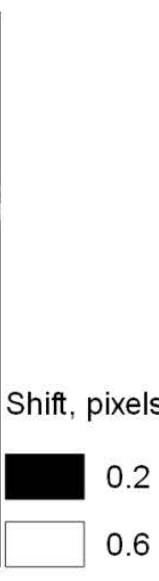

0.6

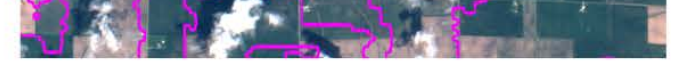

(c)

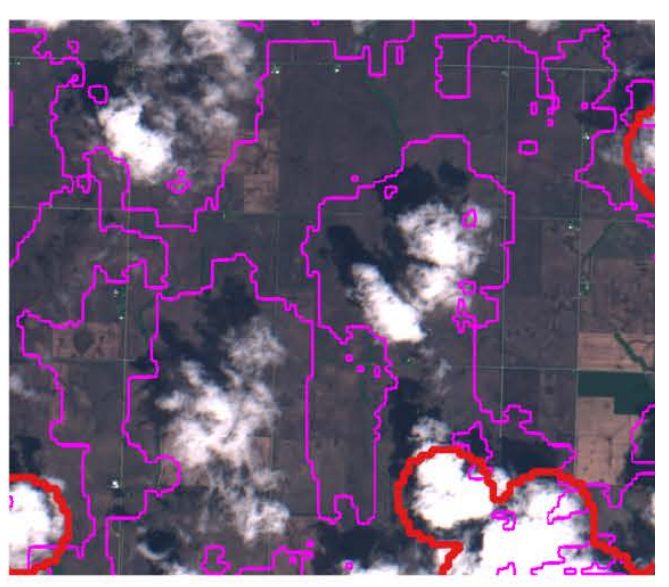

(f)

Example of cloud detection for Sentinel-2A/MSI images acquired over the US (tile 16TCK) on 15 June 2016 (a) and 21 May 2017 (d). True color images (combination of bands 4, 3 and 2) at $10 \mathrm{~m}$ spatial resolution along with the built-in cloud mask (in red) are shown in subplots (a) and (d); shifts estimated from band 4 and 2 images using phase correlation are shown in (b) and (e); cloud masks (in magenta) derived from the multi-spectral misregistration using a threshold of 0.2 pixels for shifts are shown in subplots (c) and (f). 


\section{Conclusions}

- Phase correlation proved to be a robust approach that allowed us to identify 100's and 1000's of control points on Landsat-8/Sentinel-2A images acquired more than 100 days apart.

- Misregistration of up to $\mathbf{1 . 6}$ pixels at $\mathbf{3 0} \mathrm{m}$ resolution between multitemporal Landsat-8 and Sentinel-2A images, and 1.2 pixels (same orbits) and 2.8 pixels (adjacent orbits) at $10 \mathrm{~m}$ resolution between multi-temporal Sentinel-2A images were observed.

- The Random Forest regression used for constructing the mapping function showed best results, yielding an average RMSE error of $0.07 \pm$ 0.02 pixels at $30 \mathrm{~m}$, and $0.09 \pm 0.05$ at $10 \mathrm{~m}$

- Sentinel-2A multi-spectral misregistration:

- shifts of more than 1.1 pixels can be observed for moving targets such as airplanes

- sub-pixels shifts of $\mathbf{0 . 2}$ to $\mathbf{0 . 8}$ pixels are observed for clouds, and can be used for cloud detection as one of the criteria 
